\title{
CERTAIN NEW SUBCLASSES OF ANALYTIC AND BI-UNIVALENT FUNCTIONS USING SALAGEAN OPERATOR
}

\author{
TIMILEHIN GIDEON SHABA \\ Department of Mathematics, University of Ilorin, Ilorin, Nigeria \\ Corresponding author: shabatimilehin@gmail.com
}

Received July 12, 2020

\begin{abstract}
Aвstract. Bi-univalent functions in $\Delta=\{z \in \mathfrak{C}:|z|<1\}$ consisting of two new subclasses signified by $\mathfrak{M}_{\mathfrak{E}}^{\gamma, h}(\Im)$ and $\mathfrak{M}_{\mathfrak{E}}^{\gamma, h}(\mu)$ which are introduced by applying Salagean differential operator. The coefficient estimates $\left|V_{2}\right|,\left|V_{3}\right|$ and $\left|V_{4}\right|$ on the new subclasses is investigated and importance results are indicated.

2010 Mathematics Subject Classification. 30C45; 30C50.

Key words and phrases. Bi-univalent function; Univalent function; salagean operator; coefficients estimaties.
\end{abstract}

\section{INTRODUCTION}

We indicate by $\mathfrak{J}$ the subclass of class of function $\mathfrak{T}$ which is of the form

$$
\varphi(z)=z+\sum_{\varsigma=2}^{\infty} V_{\varsigma} z^{\varsigma}
$$

consisting of function which are holomorphic and univalent in unit disk $\Delta=\{z \in \mathfrak{C}:|z|<1\}$. Let $\mathfrak{S}^{*}(\rho)$ and $\mathfrak{K}(\rho)$ indicate the familiar classes of starlike and convex function of order $\rho$ $(0 \leq \rho<1)$ respectively (see [5]).

Let $\varphi^{-1}(z)$ be the inverse of the function $\varphi(z)$ then we have

$$
\begin{gathered}
\varphi^{-1}(\varphi(z))=z, \\
\varphi\left(\varphi^{-1}(l)\right)=l, \quad|l|<r_{0}(\varphi) ; r_{0}(\varphi) \geq \frac{1}{4}
\end{gathered}
$$

DOI: 10.28924/APJM/7-29 
therefore,

$$
\varphi^{-1}(l)=\eta(l)=l-V_{2} l^{2}+\left(2 V_{2}^{2}-V_{3}\right) l^{3}-\left(5 V_{2}^{3}-5 V_{2} V_{3}+V_{4}\right) l^{4}+\cdots
$$

A function $\varphi(z) \in \mathfrak{T}$ denoted by $\mathfrak{E}$ is said to be bi-univalent in $\Delta$, considering that $\varphi(z)$ and $\varphi^{-1}(z)$ are univalent in $\Delta,($ for more details see; [10], [22], [4], [1], [12], [3], [16], [21], [23], [15]). Also various researches ( [6], [19], [11], [13], [14], [9], [8]) obtain the coefficient $\left|V_{2}\right|$ and $\left|V_{3}\right|$ of bi-univalent function for different subclass of the function class $\mathfrak{E}$. For $\varphi(z) \in \mathfrak{T}$, Salagean [18] introduced the differential operator $\mathfrak{D}^{h}$ which is defined by

$$
\begin{gathered}
\mathfrak{D}^{0} \varphi(z)=\varphi(z) ; \\
\mathfrak{D}^{1} \varphi(z)=\mathfrak{D} \varphi(z)=z \varphi^{\prime}(z) ; \\
\mathfrak{D}^{h} \varphi(z)=\mathfrak{D}\left(\mathfrak{D}^{h-1} \varphi(z)\right) .
\end{gathered}
$$

then,

$$
\mathfrak{D}^{h} \varphi(z)=z+\sum_{\varsigma=2}^{\infty} \varsigma^{h} V_{\varsigma} z^{\varsigma}
$$

where $h \in \mathcal{N}_{0}=\mathcal{N} \cup\{0\}=0,1,2,3, \cdots$

Definition 1.1. [2] Let $\varphi(z) \in \mathcal{L}$, suppose $0 \leq \rho<1$ and $\gamma \geq 1$ is real. Then $\psi(z) \in L_{\gamma}(\rho)$ of $\gamma$-pseodu-starlike function of order $\rho$ in $\Delta$ if and only if

$$
\Re\left(\frac{z\left[\varphi^{\prime}(z)\right]^{\gamma}}{\varphi(z)}\right)>\rho .
$$

Babalola [2] verified that, all pseodu-starlike function are Bazilevic of type $\left(1-\frac{1}{\gamma}\right)$, order $\rho^{\frac{1}{\gamma}}$ and univalent in $\Delta$.

This study is inspired by the earlier work of Girgaonkar et al. [7], thus we introduce two new subclasses $\mathfrak{M}_{\mathfrak{E}}^{\gamma, h}(\Im)$ and $\mathfrak{M}_{\mathfrak{E}}^{\gamma, h}(\mu)$ of the function class $\mathfrak{E}$ associated with Salagean differential operator and determine the improved estimates on the initial coefficient $\left|V_{2}\right|,\left|V_{3}\right|$ and $\left|V_{4}\right|$ for the functions in these subclasses.

Lemma 1.1. [17] If $r(z) \in \mathcal{P}$ and $z \in \nabla$, then $\left|w_{n}\right| \leq 2$ for each $n$. where $\mathcal{P}$ is the family of all function $r$ analytic in $\Delta$ for which $\Re(r(z))>0$,

$$
r(z)=1+w_{1} z+w_{2} z^{2}+\cdots
$$

where $z \in \Delta$. 
2. Coefficient bounds for the Function Class $\mathfrak{M}_{\mathfrak{E}}^{\gamma, h}(\Im)$

Definition 2.1. A function $\varphi(z) \in \mathfrak{T}$ is in the class $\mathfrak{M}_{\mathfrak{E}}^{\gamma, h}(\Im)$ if the following condition are fulfilled:

$$
\left|\arg \left[\left(\left(D^{h} \varphi(z)\right)^{\prime}\right)^{\gamma}\right]\right|<\frac{\Im \pi}{2} \quad z \in \nabla
$$

and

$$
\left|\arg \left[\left(\left(D^{h} \eta(l)\right)^{\prime}\right)^{\gamma}\right]\right|<\frac{\Im \pi}{2} \quad l \in \nabla,
$$

where $\varphi(z) \in \mathfrak{E}, \gamma>0,0<\Im \leq 1$ and

$$
\eta(l)=l-V_{2} l^{2}+\left(2 V_{2}^{2}-V_{3}\right) l^{3}-\left(5 V_{2}^{3}-5 V_{2} V_{3}+V_{4}\right) l^{4}+\cdots
$$

\section{Remark 2.1. .}

(1) $\mathfrak{M}_{\mathfrak{E}}^{1,0}(\Im)=\mathfrak{M}_{\mathfrak{E}}(\Im)$ which Srivastava et al. [20] introduced and studied.

(2) $\mathfrak{M}_{\mathfrak{E}}^{\gamma, 0}(\Im)=\mathfrak{M}_{\mathfrak{E}}^{\gamma}(\Im)$ which Girgaonkar et al. [7] introduced and studied.

Now we have the following theorem and the proof.

Theorem 2.1. A function $\varphi(z) \in \mathfrak{T}$ is in the class $\mathfrak{M}_{\mathfrak{E}}^{\gamma, h}(\Im)$. Then

$$
\begin{gathered}
\left|V_{2}\right| \leq \frac{\sqrt{2} \Im}{\sqrt{2^{h} \gamma^{2}\left(2^{h+1}-2^{h} \Im+\Im\right)-\gamma \Im\left(2^{2 h+1}-3^{h+1}\right)}}, \\
\left|V_{3}\right| \leq \frac{3^{h+1} \Im^{2}+2^{2 h+1} \Im \gamma}{3^{h+1} 2^{2 h} \gamma^{2}}
\end{gathered}
$$

and

$$
\begin{aligned}
&\left|V_{4}\right| \leq \frac{\Im}{2^{2 h+1} \gamma} {\left[1+2(\Im-1)\left(1+\frac{(\Im-2)}{3}\right)\right] } \\
&+ \frac{\sqrt{2} \Im^{2}}{\sqrt{2^{h} \gamma^{2}\left(2^{h+1}-2^{h} \Im+\Im\right)-\gamma \Im\left(2^{2 h+1}-3^{h+1}\right)}}\left[\frac{5}{3^{h+1} \gamma}+\right. \\
&\left.\frac{3^{h+1}(1-\gamma) \Im}{\left.2^{2 h+1} \gamma^{2}\left(2^{h+1}-2^{h \Im}+\Im\right)-2^{h+1} \gamma \Im\left(2^{2 h+1}-3^{h+1}\right)\right]}\right]
\end{aligned}
$$

Proof. From (2.1) and (2.2) we have,

$$
\left(\left(D^{h} \varphi(z)\right)^{\prime}\right)^{\gamma}=[y(z)]^{\Im}
$$

and

$$
\left(\left(D^{h} \eta(l)\right)^{\prime}\right)^{\gamma}=[x(l)]^{\Im}
$$


where $y(z)$ and $x(l)$ are in the class $\mathcal{P}$ which is of the form

$$
y(z)=1+y_{1} z+y_{2} z^{2}+y_{3} z^{3}+\cdots
$$

$$
x(l)=1+x_{1} l+x_{2} l^{2}+x_{3} l^{3}+\cdots
$$

\section{Hence,}

$$
\begin{array}{r}
{[y(z)]^{\Im}=1+\Im y_{1} z+\left(\Im y_{2}+\frac{\Im(\Im-1) y_{1}^{2}}{2 !}\right) z^{2}+\left(\Im y_{3}+\Im(\Im-1) y_{1} y_{2}\right.} \\
\left.+\frac{\Im(\Im-1)(\Im-2)}{3 !} y_{1}^{3}\right) z^{3}+\cdots \\
{[x(l)]^{\Im}=1+\Im x_{1} l+\left(\Im x_{2}+\frac{\Im(\Im-1) x_{1}^{2}}{2 !}\right) l^{2}+\left(\Im x_{3}+\Im(\Im-1) x_{1} x_{2}\right.} \\
\left.+\frac{\Im(\Im-1)(\Im-2)}{3 !} x_{1}^{3}\right) l^{3}+\cdots
\end{array}
$$

Now, equating the coefficient in (2.6) and (2.7) we get

$$
\begin{gathered}
2^{h+1} \gamma V_{2}=\Im y_{1} \\
3^{h+1} \gamma V_{3}+2^{2 h+1} V_{2}^{2} \gamma(\gamma-1)=\Im y_{2}+\frac{\Im(\Im-1) y_{1}^{2}}{2 !} \\
4^{h+1} \gamma V_{4}+2^{h+1} 3^{h+2} \gamma(\gamma-1) V_{2} V_{3}=\Im y_{3}+\Im(\Im-1) y_{1} y_{2}+\frac{\Im(\Im-1)(\Im-2)}{3 !} y_{1}^{3} \\
-2^{h+1} \gamma V_{2}=\Im x_{1} \\
2 \gamma\left[3^{h+1}-2^{2 h}+2^{2 h} \gamma\right] V_{2}^{2}-3^{h+1} \gamma V_{3}=\Im x_{2}+\frac{\Im(\Im-1) x_{1}^{2}}{2 !}
\end{gathered}
$$




$$
\begin{array}{r}
-4^{g+1} \gamma V_{4}-\left(5 \cdot 4^{h+1}+2^{h+1} 3^{h+1} \gamma-2^{h+2} 3^{h+1}\right) V_{2}^{3} \gamma+V_{2} V_{3} \gamma\left(5 \cdot 4^{h+1}+2^{h+1} 3^{h+1} \gamma\right. \\
\left.-2^{h+1} 3^{h+1}\right)=\Im x_{3}+\Im(\Im-1) x_{1} x_{2}+\frac{\Im(\Im-1)(\Im-2)}{3 !} x_{1}^{3}
\end{array}
$$

From (2.10) and (2.13) we obtain

$$
y_{1}=-x_{1}
$$

and

$$
2^{2 h+3} \gamma^{2} V_{2}^{2}=\Im^{2}\left(y_{1}^{2}+x_{1}^{2}\right)
$$

Now, adding (2.11) and (2.14), we have

$$
\left[2 \gamma^{2}\left(2^{2 h}+2^{h}\right)-2 \gamma\left(2^{2 h+1}-3^{h+1}\right)\right] V_{2}^{2}=\Im\left(y_{2}+x_{2}\right)+\frac{\Im(\Im-1)}{2 !}\left(y_{1}^{2}+x_{1}^{2}\right)
$$

from (2.17), we have

$$
V_{2}^{2}=\frac{\Im^{2}\left(y_{2}+x_{2}\right)}{2^{h+1} \gamma^{2}\left(2^{h+1}-2^{h} \Im+\Im\right)-2 \gamma \Im\left(2^{2 h+1}-3^{h+1}\right)}
$$

Applying lemma (1.1), we get

$$
\left|V_{2}\right| \leq \frac{\sqrt{2} \Im}{\sqrt{2^{h} \gamma^{2}\left(2^{h+1}-2^{h} \Im+\Im\right)-\gamma \Im\left(2^{2 h+1}-3^{h+1}\right)}},
$$

Now, to get the bound $\left|V_{3}\right|$, we subtract (2.14) from (2.11) to have

$$
\begin{aligned}
& 2.3^{h+1} \gamma V_{3}+2^{2 h+1} V_{2}^{2} \gamma(\gamma-1)-2 \gamma\left[3^{h+1}-2^{2 h}+2^{2 h} \gamma\right] V_{2}^{2} \\
& =\Im\left(y_{2}-x_{2}\right) \\
& +\frac{\Im(\Im-1)}{2 !}\left(y_{1}^{2}-x_{1}^{2}\right)
\end{aligned}
$$

then from (2.16), we have

$$
\begin{gathered}
2.3^{h+1} \gamma V_{3}+2^{2 h+1} V_{2}^{2} \gamma(\gamma-1)-2 \gamma\left[3^{h+1}-2^{2 h}+2^{2 h} \gamma\right] V_{2}^{2}=\Im\left(y_{2}-x_{2}\right) \\
V_{3}=V_{2}^{2}+\frac{\Im\left(y_{2}-x_{2}\right)}{2.3^{h+1} \gamma} \\
V_{3}=\frac{\Im^{2} y_{1}^{2}}{2^{2 h+2} \gamma^{2}}+\frac{\Im\left(y_{2}-x_{2}\right)}{2.3^{h+1} \gamma}
\end{gathered}
$$


Applying lemma (1.1), we get

$$
\left|V_{3}\right| \leq \frac{\Im^{2}}{2^{2 h} \gamma^{2}}+\frac{2 \Im}{3^{h+1} \gamma}
$$

$$
\left|V_{3}\right| \leq \frac{3^{h+1} \Im^{2}+2^{2 h+1} \Im \gamma}{3^{h+1} 2^{2 h} \gamma^{2}}
$$

Subtracting (2.15) from (2.12) we obtain the bound on $\left|V_{4}\right|$

$$
\begin{array}{r}
4^{h+1} \gamma V_{4}+2^{h+1} 3^{h+2} \gamma(\gamma-1) V_{2} V_{3}+4^{g+1} \gamma V_{4}+\left(5 \cdot 4^{h+1}+2^{h+1} 3^{h+1} \gamma-2^{h+2} 3^{h+1}\right) V_{2}^{3} \gamma \\
-V_{2} V_{3} \gamma\left(5 \cdot 4^{h+1}+2^{h+1} 3^{h+1} \gamma-2^{h+1} 3^{h+1}\right)=\Im\left(x_{3}-y_{3}\right)+\Im(\Im-1)\left(x_{1} x_{2}-y_{1} y_{2}\right) \\
+\frac{\Im(\Im-1)(\Im-2)}{3 !}\left(x_{1}^{3}-y_{1}^{3}\right) \\
2.4^{h+1} \gamma V_{4}+V_{2}\left[5 \cdot 4^{h+1} V_{2}^{2} \gamma+2^{h+2} 3^{h+1} \gamma(\gamma-1) V_{2}^{2}-5 \cdot 4^{h+1} V_{3} \gamma\right]=\Im\left(x_{3}-y_{3}\right) \\
+\Im(\Im-1)\left(x_{1} x_{2}-y_{1} y_{2}\right)+\frac{\Im(\Im-1)(\Im-2)}{3 !}\left(x_{1}^{3}-y_{1}^{3}\right)
\end{array}
$$

from (2.22), we get

$$
\begin{aligned}
2.4^{h+1} \gamma V_{4}-V_{2}\left[\frac{5 \cdot 4^{h+1} \gamma \Im\left(y_{2}-x_{2}\right)}{2.3^{h+1} \gamma}\right. & \left.+2^{h+2} 3^{h+1} \gamma(1-\gamma) V_{2}^{2}\right]=\Im\left(x_{3}-y_{3}\right) \\
& +\Im(\Im-1)\left(x_{1} x_{2}-y_{1} y_{2}\right)+\frac{\Im(\Im-1)(\Im-2)}{3 !}\left(x_{1}^{3}-y_{1}^{3}\right)
\end{aligned}
$$

also from (2.19). we have

$$
\begin{array}{r}
2.4^{h+1} \gamma V_{4}=\frac{\sqrt{2} \Im}{\sqrt{2^{h} \gamma^{2}\left(2^{h+1}-2^{h} \Im+\Im\right)-\gamma \Im\left(2^{2 h+1}-3^{h+1}\right)}}\left[\frac{5 \cdot 4^{h+1} \gamma \Im\left(y_{2}-x_{2}\right)}{2.3^{h+1} \gamma}\right. \\
\left.+2^{h+2} 3^{h+1} \gamma(1-\gamma)\left(\frac{\Im^{2}\left(y_{2}+x_{2}\right)}{2^{h+1} \gamma^{2}\left(2^{h+1}-2^{h} \Im+\Im\right)-2 \gamma \Im\left(2^{2 h+1}-3^{h+1}\right)}\right)\right]+\Im\left(x_{3}-y_{3}\right) \\
+\Im(\Im-1)\left(x_{1} x_{2}-y_{1} y_{2}\right)+\frac{\Im(\Im-1)(\Im-2)}{3 !}\left(x_{1}^{3}-y_{1}^{3}\right)
\end{array}
$$


Applying lemma (1.1), we get

$$
\begin{aligned}
\left|V_{4}\right| \leq \frac{\Im}{2^{2 h+1} \gamma}\left[1+2(\Im-1)\left(1+\frac{(\Im-2)}{3}\right)\right] & \sqrt{2} \Im^{2} \\
+ & \frac{3^{h+1}(1-\gamma) \Im}{\sqrt{2^{h} \gamma^{2}\left(2^{h+1}-2^{h} \Im+\Im\right)-\gamma \Im\left(2^{2 h+1}-3^{h+1}\right)}} \\
& {\left[\frac{5}{3^{h+1} \gamma}+\frac{5}{\left.2^{2 h+1} \gamma^{2}\left(2^{h+1}-2^{h} \Im+\Im\right)-2^{h+1} \gamma \Im\left(2^{2 h+1}-3^{h+1}\right)\right]}\right] }
\end{aligned}
$$

3. Coefficient bounds for the Function Class $\mathfrak{M}_{\mathfrak{E}}^{\gamma, h}(\mu)$

Definition 3.1. A function $\varphi(z) \in \mathfrak{T}$ is in the class $\mathfrak{K}_{\mathfrak{E}}^{\phi, g}(\mu)$ if the following condition are fulfilled:

$$
\Re\left[\left(\left(D^{h} \varphi(z)\right)^{\prime}\right)^{\gamma}\right]>\mu \quad z \in \nabla,
$$

and

$$
\Re\left[\left(\left(D^{h} \eta(l)\right)^{\prime}\right)^{\gamma}\right]>\mu \quad l \in \nabla,
$$

where $\varphi(z) \in \mathfrak{E}, \gamma>0,0 \leq \mu<1$ and

$$
\eta(l)=l-V_{2} l^{2}+\left(2 V_{2}^{2}-V_{3}\right) l^{3}-\left(5 V_{2}^{3}-5 V_{2} V_{3}+V_{4}\right) l^{4}+\cdots
$$

\section{Remark 3.1. .}

(1) $\mathfrak{M}_{\mathfrak{E}}^{1,0}(\mu)=\mathfrak{K}_{\mathfrak{E}}(\mu)$ which Srivastava et al. [20] introduced and studied.

(2) $\mathfrak{M}_{\mathfrak{E}}^{\gamma, 0}(\mu)=\mathfrak{M}_{\mathfrak{E}}^{\gamma}(\mu)$ which Girgaonkar et al. [7] introduced and studied.

Now we have the following theorem and the proof.

Theorem 3.1. A function $\varphi(z) \in \mathfrak{T}$ is in the class $\mathfrak{M}_{\mathfrak{E}}^{\gamma, h}(\mu)$. Then

$$
\left|V_{2}\right| \leq \sqrt{\frac{2(1-\mu)}{\gamma^{2}\left(2^{2 h}+2^{h}\right)-\gamma\left(2^{2 h+1}-3^{3 h+1}\right)}},
$$

$$
\left|V_{3}\right| \leq \frac{(1-\mu)\left[2^{2 h+1} \gamma-3^{h+1} \mu+3^{2 h+1}\right]}{2^{2 h} 3^{h+1} \gamma^{2}}
$$


and

$$
\begin{array}{r}
\left|V_{4}\right| \leq \frac{(1-\mu)}{2^{2 h+1} \gamma}+\sqrt{\frac{2(1-\mu)}{\gamma^{2}\left(2^{2 h}+2^{h}\right)-\gamma\left(2^{2 h+1}-3^{3 h+1}\right)}\left[\frac{5(1-\mu)}{3^{h+1} \gamma}\right.} \\
\left.+\frac{3^{h+1}(1-\mu)(1-\gamma)}{2^{h+1}\left(\gamma^{2}\left(2^{2 h}+2^{h}\right)-\gamma\left(2^{2 h+1}-3^{3 h+1}\right)\right)}\right]
\end{array}
$$

Proof. From (3.1) and (3.2) we have,

$$
\left(\left(D^{h} \varphi(z)\right)^{\prime}\right)^{\gamma}=\mu+(1-\mu) y(z)
$$

and

$$
\left(\left(D^{h} \eta(l)\right)^{\prime}\right)^{\gamma}=\mu+(1-\mu) x(l)
$$

where $y(z)$ and $x(l)$ in $\mathcal{P}$ given by (2.8) and (2.9), that is

$$
\mu+(1-\mu) y(z)=1+(1-\mu) y_{1} z+(1-\mu) y_{2} z^{2}+(1-\mu) y_{3} z^{3} \cdots
$$

and

$$
\mu+(1-\mu) x(l)=1+(1-\mu) x_{1} l+(1-\mu) x_{2} l^{2}+(1-\mu) x_{3} l^{3}+\cdots
$$

Equating the coefficients of (3.6) and (3.7) we get

$$
\begin{gathered}
2^{h+1} \gamma V_{2}=(1-\mu) y_{1} \\
3^{h+1} \gamma V_{3}+2^{2 h+1} V_{2}^{2} \gamma(\gamma-1)=(1-\mu) y_{2} \\
4^{h+1} \gamma V_{4}+2^{h+1} 3^{h+2} \gamma(\gamma-1) V_{2} V_{3}=(1-\mu) y_{3} \\
-2^{h+1} \gamma V_{2}=(1-\mu) x_{1}, \\
2 \gamma\left[3^{h+1}-2^{2 h}+2^{2 h} \gamma\right] V_{2}^{2}-3^{h+1} \gamma V_{3}=(1-\mu) x_{2} \\
-4^{g+1} \gamma V_{4}-\left(5 \cdot 4^{h+1}+2^{h+1} 3^{h+1} \gamma-2^{h+2} 3^{h+1}\right) V_{2}^{3} \gamma+V_{2} V_{3} \gamma\left(5 \cdot 4^{h+1}\right. \\
\left.+2^{h+1} 3^{h+1} \gamma-2^{h+1} 3^{h+1}\right)=(1-\mu) x_{3}
\end{gathered}
$$


From (3.8) and (3.11) we get

$$
y_{1}=-x_{1}
$$

and

$$
2^{2 h+3} \gamma^{2} V_{2}^{2}=(1-\mu)^{2}\left(y_{1}^{2}+x_{1}^{2}\right)
$$

Now, adding (3.9) and (3.12), we have

$$
\left[2 \gamma^{2}\left(2^{2 h}+2^{h}\right)-2 \gamma\left(2^{2 h+1}-3^{h+1}\right)\right] V_{2}^{2}=(1-\mu)\left(y_{2}+x_{2}\right)
$$

$$
\left|V_{2}^{2}\right| \leq \frac{(1-\mu)\left(\left|y_{2}\right|+\left|x_{2}\right|\right)}{\left[2 \gamma^{2}\left(2^{2 h}+2^{h}\right)-2 \gamma\left(2^{2 h+1}-3^{h+1}\right)\right]}
$$

Applying Lemma 1.1, we get

$$
\left|V_{2}\right| \leq \sqrt{\frac{2(1-\mu)}{\gamma^{2}\left(2^{2 h}+2^{h}\right)-\gamma\left(2^{2 h+1}-3^{3 h+1}\right)}},
$$

Now, to get the bound $\left|V_{3}\right|$, we subtract (3.12) from (3.9) to have

$$
\begin{gathered}
2.3^{h+1} \gamma V_{3}+2^{2 h+1} V_{2}^{2} \gamma(\gamma-1)-2 \gamma\left[3^{h+1}-2^{2 h}+2^{2 h} \gamma\right] V_{2}^{2}=(1-\mu)\left(y_{2}-x_{2}\right) \\
V_{3}=V_{2}^{2}+\frac{(1-\mu)\left(y_{2}-x_{2}\right)}{2.3^{h+1} \gamma} \\
V_{3}=\frac{2(1-\mu)^{2} y_{1}^{2}}{2^{2 h+3} \gamma^{2}}+\frac{(1-\mu)\left(y_{2}-x_{2}\right)}{2 \cdot 3^{h+1} \gamma}
\end{gathered}
$$

Applying Lemma 1.1, we get

$$
\left|V_{3}\right| \leq \frac{(1-\mu)\left[2^{2 h+1} \gamma-3^{h+1} \mu+3^{2 h+1}\right]}{2^{2 h} 3^{h+1} \gamma^{2}}
$$

Subtracting (3.13) from (3.10) we obtain the bound on $\left|V_{4}\right|$

$$
\begin{gathered}
4^{h+1} \gamma V_{4}+2^{h+1} 3^{h+2} \gamma(\gamma-1) V_{2} V_{3}+4^{g+1} \gamma V_{4}+\left(5 \cdot 4^{h+1}+2^{h+1} 3^{h+1} \gamma-2^{h+2} 3^{h+1}\right) \\
V_{2}^{3} \gamma-V_{2} V_{3} \gamma\left(5 \cdot 4^{h+1}+2^{h+1} 3^{h+1} \gamma-2^{h+1} 3^{h+1}\right)=(1-\mu)\left(y_{3}-x_{3}\right) \\
2.4^{h+1} \gamma V_{4}+V_{2}\left[5 \cdot 4^{h+1} V_{2}^{2} \gamma+2^{h+2} 3^{h+1} \gamma(\gamma-1) V_{2}^{2}-5 \cdot 4^{h+1} V_{3} \gamma\right]=(1-\mu)\left(y_{3}-x_{3}\right)
\end{gathered}
$$


from (3.18), we have

$$
\begin{aligned}
2.4^{h+1} \gamma\left|V_{4}\right| \leq(1-\mu)\left(y_{3}-x_{3}\right)+\sqrt{\frac{(1-\mu)\left(\left|y_{2}\right|+\left|x_{2}\right|\right)}{\left[2 \gamma^{2}\left(2^{2 h}+2^{h}\right)-2 \gamma\left(2^{2 h+1}-3^{h+1}\right)\right]}} & \\
& {\left[\frac{5 \cdot 4^{h+1} \gamma(1-\mu)\left(y_{2}-x_{2}\right)}{2.3^{h+1} \gamma}+2^{h+2} 3^{h+1} \gamma(1-\gamma)\right.} \\
& {\left.\left[\frac{(1-\mu)\left(\left|y_{2}\right|+\left|x_{2}\right|\right)}{\left[2 \gamma^{2}\left(2^{2 h}+2^{h}\right)-2 \gamma\left(2^{2 h+1}-3^{h+1}\right)\right]}\right]\right] }
\end{aligned}
$$

Applying Lemma 1.1, we get

$$
\begin{gathered}
\left|V_{4}\right| \leq \frac{(1-\mu)}{2^{2 h+1} \gamma}+\sqrt{\frac{2(1-\mu)}{\gamma^{2}\left(2^{2 h}+2^{h}\right)-\gamma\left(2^{2 h+1}-3^{3 h+1}\right)}} \\
{\left[\frac{5(1-\mu)}{3^{h+1} \gamma}+\frac{3^{h+1}(1-\mu)(1-\gamma)}{2^{h+1}\left(\gamma^{2}\left(2^{2 h}+2^{h}\right)-\gamma\left(2^{2 h+1}-3^{3 h+1}\right)\right)}\right]}
\end{gathered}
$$

Acknowledgment. The author thank the referees(s) for their relevant contributions which improved this research.

\section{REFERENCES}

[1] A. Aldawish, T. Al-Hawary and B.A. Frasin, Subclasses of bi-univalent function defined by Frasin differential operator, Mathematics. 8 (2020), 783.

[2] K.O. Babalola, On $\lambda$-pseudo-starlike function, J. Class. Anal. 3 (2013), 137-147.

[3] D.A. Brannan and T.S. Taha, On some classes of bi-univalent functions, Studia. Univ. Babes-Bolyai Math. 31 (1986), 70-77.

[4] D.A. Brannan, J. Clunie and W.E. Kirwan, Coefficient estimate for a class of starlike functions, Canad. J. Math. 22 (2009), 476-485.

[5] P.L. Duren, Univalent functions, Grundlehren der Mathematischen Wissenschaften, Springer, New York, (2004).

[6] B.A. Frasin and M.K. Aouf, New subclasses of bi-univalent functions, Appl. Math. Lett. 24 (2004), 1529-1573.

[7] V.B. Girgaonkar, S.B. Joshi and P.P. Yadav, Certain special subclasses of analytic function associated with bi-univalent functions , Palestine J. Math. 6(2) (2017), 617-623.

[8] S.B. Joshi, and P.P. Yadav, Coefficient bounds for new subclasses of bi-univalent function associated with pseudo-starlike functions, Ganita J. 69 (2019), 67-74.

[9] J. Jothibasu, Certain subclasses of bi-univalent functions defined by salagean operator, Elec. J. Math. Anal. Appl. 3 (2015), 150-157. 
[10] M. Lewin, On a coefficients problem of bi-univalent functions, Proc. Am. Math. Soc. 18 (1967), 63-68.

[11] N. Mageh, T. Rosy and S. Varma, Coefficient estimate problem for a new sunclass of bi-univalent functions, J. Complex Anal. 2013 (2013), 474231.

[12] G. Murugusundaramoorthy, N. Magesh and V. Prameela, Coefficient bounds for certain subclasses of bi-univalent functions, Abstr. Appl. Anal. 2013 (2013), 573017.

[13] G. Murugusundaramoorthy, C. Selvaraj and O. S. Badu, Coefficient estimates for pascu-type subclasses of bi-univalent function based on subordination, Int. J. Nonlinear Sci. 19(2015), 47-52.

[14] U.H. Naik and A.B. Patil, On initial coefficient inequalities for certain new subclasses of bi-univalent functions, J. Egypt. Math. Soc. 25 (2017), 291-293.

[15] E. Netanyahu, The minimal distance of the image boundary from the origin and the second coefficient of univalent function in $|z|<1$, Proc. Arch. Ration. Mech. Anal. 32 (1969), 100-112.

[16] S.0. Olatunji, and P.T. Ajayi, On subclasses of bi-univalent functions of Bazelevic type involving linear salagean operator, Int. J. Pure. Appl. Math. 92 (2014), 645-656.

[17] C.H. Pommerenke, Univalent Functions, Vandendoeck and Rupercht, Gottingen, (1975).

[18] G.S. Salagean, Subclasses of Univalent functions, Lecture Notes in Math. Spinger Verlag, Berlin. 1013 (1983), 362-372.

[19] T.G. Shaba, A. A. Ibrahim and A. A. Jimoh, On a new subclass of bi-pseudo-starlike functions defined by frasin differential operator, Adv. Math. Sci. J. 9 (7) (2020), 4829-4841.

[20] H.M. Srivastava, A. K. Mishra and P. Gochhayat, Certain subclasses of analytic and bi-univalent functions, Appl. Math. Lett. 23 (2010), 1188-1192.

[21] H.M. Srivastava and D. Bansal, Coefficient estimates for a subclassof analytic and bi-univalent functions, J. Egypt. Math. Soc. 23 (2015), 242-246

[22] T.S. Taha, Topics in univalent functions theory, Ph.D. Thesis, University of London, London, UK, (1998).

[23] Q.H. Xu, Y.C. Gui and H.M. Srivastava, A certain general subclass of analytic and bi-univalent functions and associated coefficient estimate problems, Appl. Math. Comput. 218 (2012), 11461-11465. 